\title{
Efficacy of extracorporeal shock wave treatment in calcaneal enthesophytosis
}

\author{
R Cosentino, P Falsetti, S Manca, R De Stefano, E Frati, B Frediani, F Baldi, E Selvi, \\ R Marcolongo
}

\begin{abstract}
Objective-To evaluate the efficacy of extracorporeal shock wave treatment (ESWT) in calcaneal enthesophytosis. Methods-60 patients ( 43 women, 17 men) were examined who had talalgia associated with heel spur. A single blind randomised study was performed in which 30 patients underwent a regular treatment (group 1) and 30 a simulated one (shocks of $0 \mathrm{~mJ} / \mathrm{mm}^{2}$ energy were applied) (group 2). Variations in symptoms were evaluated by visual analogue scale (VAS). Variations in the dimension of enthesophytosis were evaluated by $x$ ray examination. Variations in the grade of enthesitis were evaluated by sonography.
\end{abstract}

Results-A significant decrease of VAS was seen in group 1. Examination by $x$ ray showed morphological modifications (reduction of the larger diameter $>1 \mathrm{~mm}$ ) of the enthesophytosis in nine $(30 \%)$ patients. Sonography did not show significant changes in the grade of enthesitis just after the end of the treatment, but a significant reduction was seen after one month. In the control group no significant decrease of VAS was seen. No modification was observed by $x$ ray examination or sonography.

Conclusion-ESWT is safe and improves the symptoms of most patients with a painful heel, it can also structurally modify enthesophytosis, and reduce inflammatory oedema.

(Ann Rheum Dis 2001;60:1064-1067)

Painful heel is a common syndrome characterised by severe pain in the inferior or posterior aspect of the heel, which is aggravated by weight bearing, becoming progressively worse and often incapacitating, with evidence of a spur in about $50 \%$ of cases. ${ }^{1}$ Until now the cause of the condition has been obscure, but numerous factors have been claimed to produce painful heel with a bony spur: functional overuse, degenerative diseases, inflammatory diseases, and metabolic diseases. ${ }^{2}$

The conservative methods of treatment usually adopted have included insole supports, injections of local anaesthesia and corticosteroids, and treatment with antiphlogistic drugs. $^{34}$

Extracorporeal shock wave treatment (ESWT) is based on the use of shock wavesthat is, microsecond pressure impulses, which, depending on the energy used, can reduce painful symptoms and fragmentation of calcific deposits. $^{15-7}$
Our study aimed at evaluating the effects of ESWT on pain levels and morphological variations in enthesophytosis and in enthesis.

\section{Patients and methods}

Sixty patients (43 women, 17 men) aged 45-68 (mean 55.6), with talalgia associated with heel spur, were examined.

Inclusion criteria were pain over the radiologically examined heel spur and unsuccessful conservative treatment (insole supports, injections of local anaesthetics and corticosteroids, treatment with analgesics and non-steroidal anti-inflammatory drugs) during the six months before referral to our hospital. All patients had used analgesic and non-steroidal anti-inflammatory drugs, 25 patients from group 1 (treatment group) and 28 patients from group 2 (control group) used insole supports, 15 patients from group 1 and 19 patients from group 2 had received injections of local anaesthetics and corticosteroids.

Exclusion criteria were arthritis (rheumatoid arthritis, spondarthritis, crystal induced arthropathies), neurological abnormalities, nerve entrapment syndrome, pregnancy, age under 18 years, infectious or tumorous diseases, skin ulcerations, and bursitis.

All patients were informed of, and consented to, the treatment methods. No other treatment or drug was used during the four weeks before the trials began or during the study period. During the periods of treatment and follow up only the use of insole supports was permitted.

Consecutive patients were randomly assigned to one of two groups in a single blind study. Group 1, comprising 30 patients (18 women, $12 \mathrm{men}$ ) with a mean duration of symptoms of 8.6 months (range 6-12), received ESWT. Group 2 was the placebo group, comprising 30 patients ( 25 women, five men) with a mean duration of symptoms of 8.2 months (range 6-12).

An ESWT system was used ("Orthima", Direx Medical System Ltd) that is characterised by an electrohydraulic shock wave source assembled on a mobile arm with a full range of movement. It is also equipped with a sonographic system with a linear array $7.5 \mathrm{MHz}$ probe. Groups 1 and 2 both received six treatments (one every 7-10 days), each treatment consisting of 1200 shocks with a frequency of 120 shocks/min; the energy density used varied from 0.03 to $0.4 \mathrm{~mJ} / \mathrm{mm}^{2}$ in group 1 and was 0 $\mathrm{mJ} / \mathrm{mm}^{2}$ in group 2 . The shocks were aimed at the enthesophytosis which was identified during sonographic examination.

Variations in the dimension of the larger diameter of enthesophytosis were evaluated by $x$ ray examination at baseline and after one 

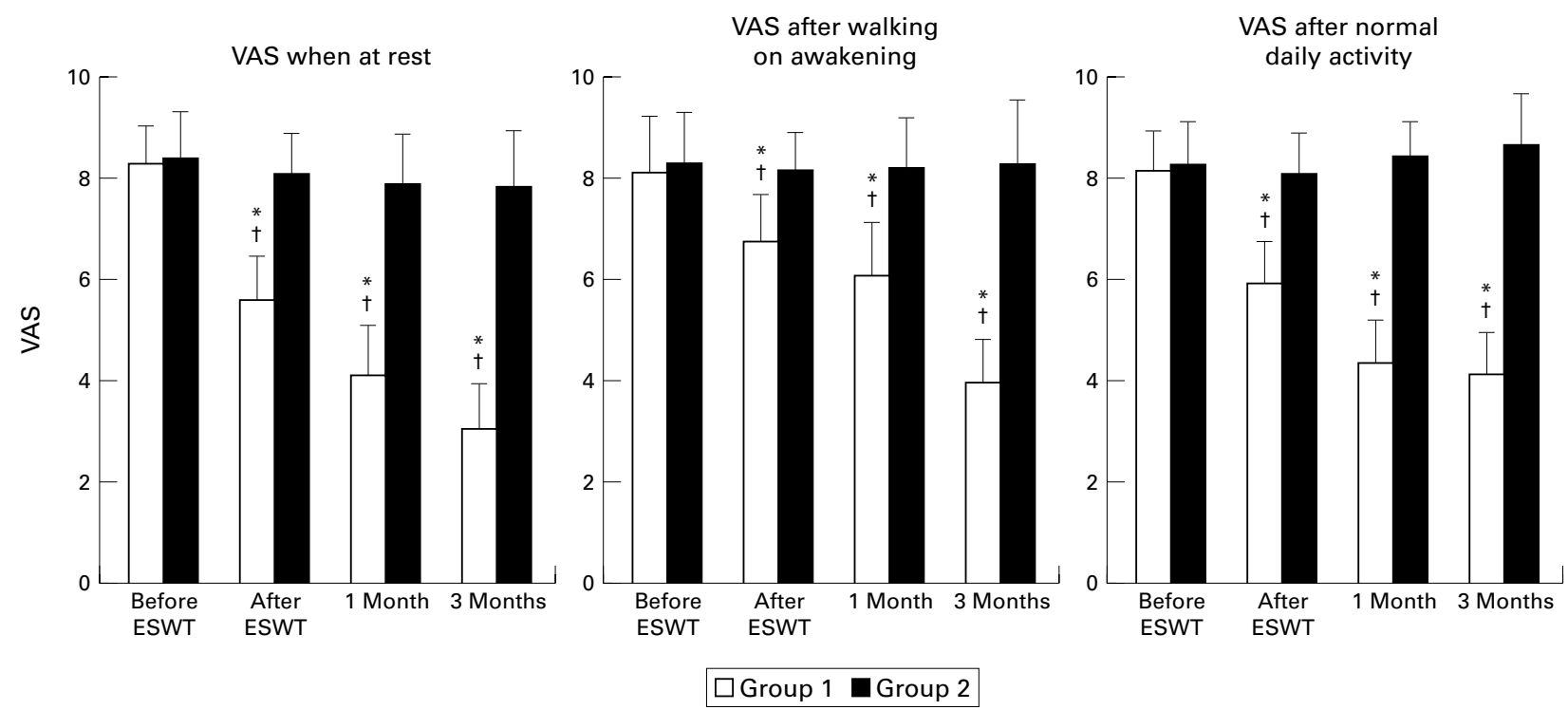

AS after normal

Figure 1 Mean visual analogue scale (VAS) score before extracorporeal shock wave treatment (ESWT), after ESWT, and one and three months later, at rest, after walking on awakening, and after normal daily activity. ${ }^{*} p<0.0001$, group 1 v group $2 ;, p<0.0001$ group 1 , baseline $v$ after ESWT, after one month, and after three months.

month from the end of the treatment (one radiologist carried out all the radiography and was not aware of the type of treatment being given to the patients). The modifications observed were classified as grade 1 (reduction in enthesophytosis $<1 \mathrm{~mm}$ ), grade 2 (reduction in enthesophytosis $>1 \mathrm{~mm}$ ).

Variations in the grade of enthesitis were evaluated by sonography on the heel before starting the treatment, just after the treatment, and one month later (one ultrasonographer carried out all the sonography and was not aware of the type of treatment being given to the patients). Ultrasound examinations were carried out with a Toshiba Tosbee Sal 240 with a linear $7.5 \mathrm{MHz}$ probe and a kitecho gel pad spacer where necessary. The following signs of enthesitis were considered: enthesophytosis, heterogeneous hypoechogenicity and swelling of enthesis, peritendinous oedema. The modifications observed were classified as grade 1 : thickening of enthesis $(<2 \mathrm{~mm}$ thicker than the contralateral asymptomatic side), heterogeneous hypoechogenicity of enthesis, and enthesophytosis; grade 2: thickening of enthesis ( $>2$ mm thicker than the contralateral asymptomatic side), heterogeneous hypoechogenicity of enthesis, and enthesophytosis; grade 3: grade 2 with peritendinous oedema. ${ }^{89}$

Pain levels were evaluated by a visual analogue scale (VAS), ranging from $0=$ no pain to $10=$ maximum pain, at rest, after walking on awakening, and after normal daily activity. Such assessment was made before, at the end of the treatment, and one and three months after the end of the treatment. The modifications observed were classified as excellent improvement (a VAS reduction of over $50 \%$ ), good improvement (a VAS reduction of between $30 \%$ and 50\%), slight improvement (a VAS reduction of between $15 \%$ and $30 \%$ ).

STATISTICS

A non-parametric Mann-Whitney U test for two independent samples was applied to compare variations of means of VAS, changes of sonographic evaluations (grading of enthesitis), and variations of the larger diameter of spurs in treated patients compared with control patients. The non-parametric Wilcoxon test for
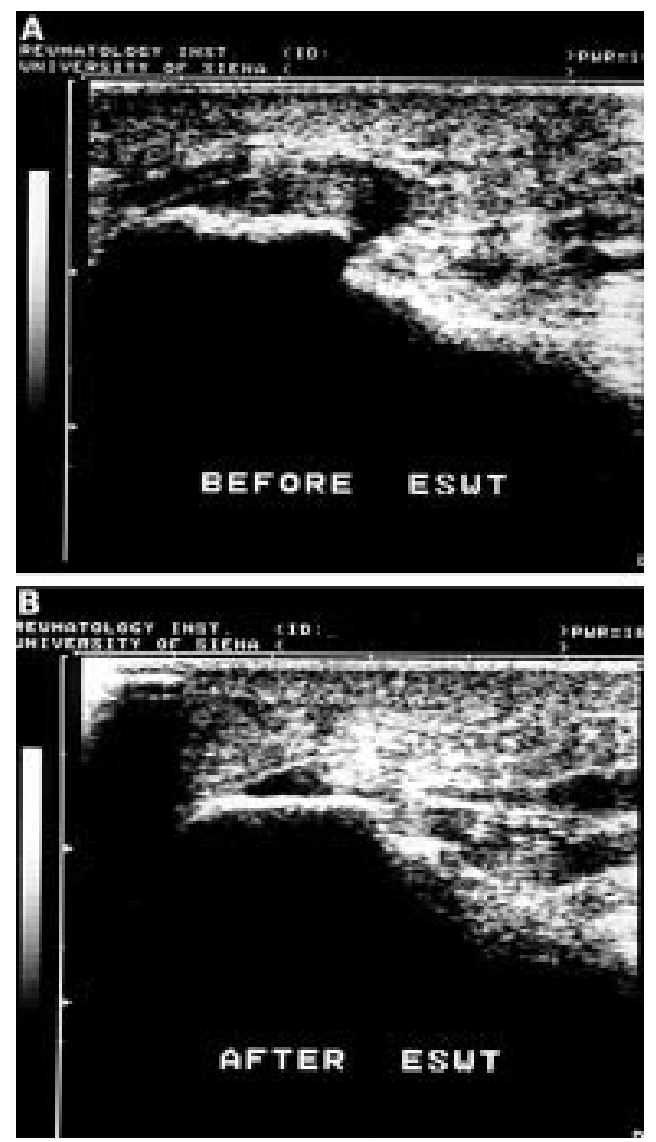

Figure 2 (A) Enthesitis of the plantar fascia (sonographic grade 3) before extracorporeal shock wave treatment (ESWT). (B) The same patient as in fig $2 A$, examined one month after the end of ESWT. An improvement of enthesitis (disappearance of the paratendinitis, reduction of thickness of the plantar fascia origin, modification of the subcalcaneal spur) can be seen. 

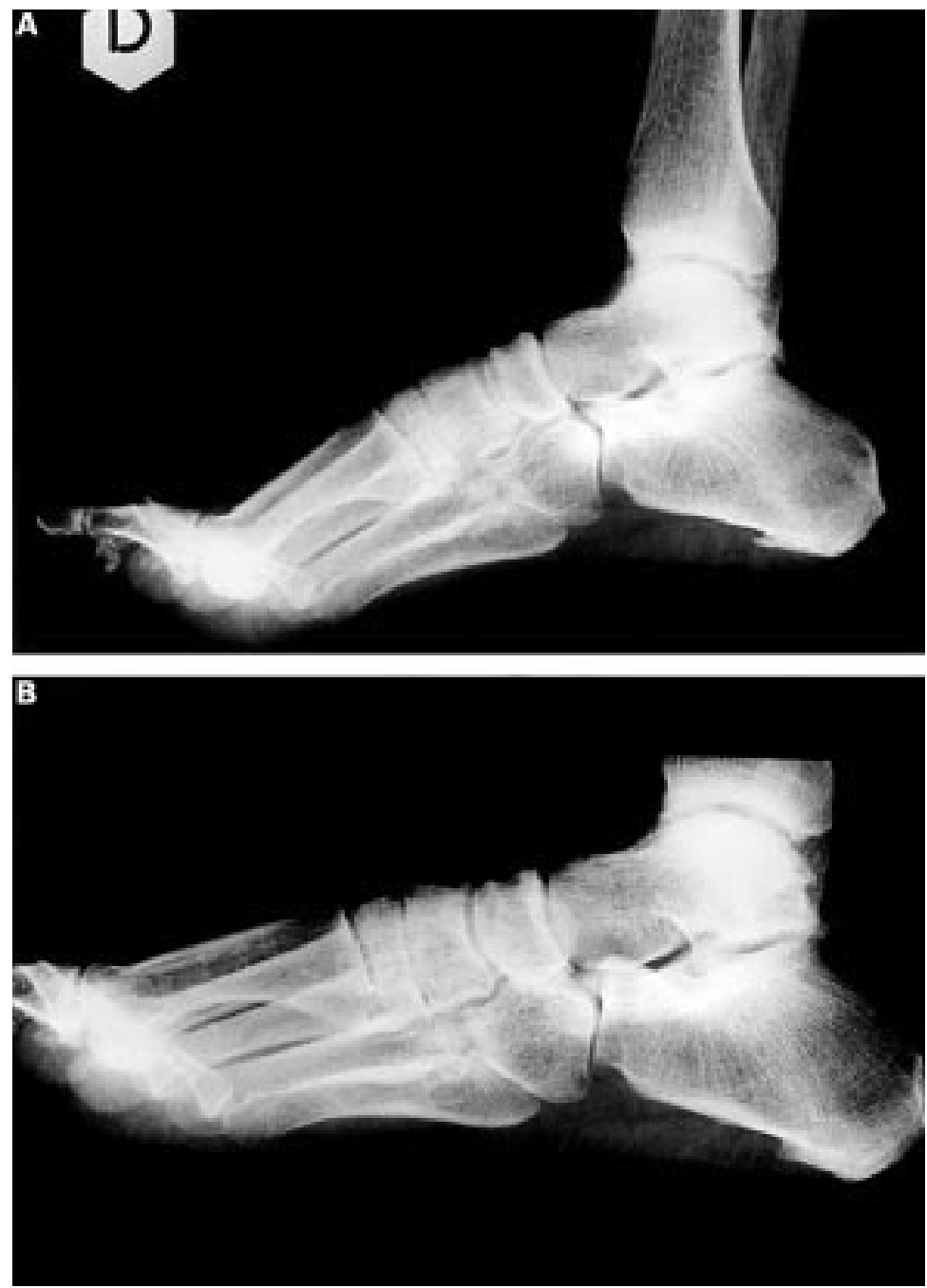

Figure 3 (A) An x ray picture of heel spur before extracorporeal shock wave treatment. (B) The same patient as in fig $3 A$ one month after the end of the treatment.

dependent samples was applied to compare means of VAS, changes of sonographic evaluations (grading of enthesitis), and variations of the larger diameter of spurs in the same group.

\section{Results}

In group 1 a significant decrease of VAS $(\mathrm{p}<0.0001)$ was seen just after the treatment and one and three months later at the three reference points - at rest, after walking on awakening, and after normal daily activity (fig 1 ). In the control group (group 2) no significant decrease of VAS $(p=0.47)$ was seen at these points (fig 1).

Sonographic evaluation in groups 1 and 2 showed no significant changes in the grade of enthesitis just after the end of the treatment, though at one month after the end of the treatment a significant decrease was seen $(p<0.001)$ in group 1 . This reduction in the grade of enthesitis was seen in $12 / 30(40 \%)$ patients in group 1, and no change was seen in $18 / 30$ $(60 \%)$ patients in this group. The decrease was significant $(p<0.01)$ compared with the control group, where the grade of enthesitis was reduced in only $2 / 30(7 \%)$ patients, worsened in $4 / 30(13 \%)$, and was unchanged in $24 / 30$ (80\%) (fig 2 ).
Examination by $x$ ray showed reduction in enthesophytosis $>1 \mathrm{~mm}$ of $9 / 30(30 \%)$ patients and reduction in enthesophytosis $<1 \mathrm{~mm}$ or no change in $21 / 30(70 \%)$ patients. Radiological reduction of enthesophytosis 1 month after the end of treatment was significant in group 1 $(\mathrm{p}<0.001)$, but not significant $(\mathrm{p}=0.068)$ in comparison with the control group (fig 3 ).

No side effects were seen. A temporary, slight redness of the skin and a transient increase in pain levels were within the norm.

\section{Discussion}

High energy extracorporeal shock waves have been used in urology for the disintegration of stone concretion for almost 15 years. ${ }^{10}$ Valchanou reported the successful application of high energy shock waves in the treatment of pseudarthrosis and delayed union of fractures, stating that the stimulation of osteogenesis achieved by shock waves can be attributed to a local cortical destruction and fragmentation and that the same effect is assumed to lead to a disintegration of calcifications in the treatment of tendinosis calcarea. ${ }^{112}$ Since 1992, low energy ESWT has been used in the treatment of pain, in particular of enthesopathy, occurring in precisely locatable areas of the locomotor apparatus. ${ }^{3}$

Loew and Jurgowski reported good results in calcifying tendinitis of the shoulder with low and high energy ${ }^{13}$; Rompe et $a l^{1}$ suggested that the pain relief observed after ESWT at energy levels comparable with those used in our study might be similar to the hyperstimulation analgesia described by Melzack. ${ }^{6}$ The positive clinical outcome of low energy ESWT for chronic plantar fasciitis has been confirmed in clinical studies. ${ }^{7}$ Maier et al reported that low energy ESWT can be regarded as an optional non-invasive therapeutic method without major side effects which can be used to avoid surgical treatment in patients with chronic courses of plantar fasciitis associated with heel spurs. ${ }^{3}$ Perlick et al also have had good results in the treatment of chronic plantar fasciitis. ${ }^{14}$

In our single blind randomised study we evaluated the efficacy of ESWT on painful heel with heel spurs and noted its effects on pain levels, on the inflammatory oedema, and on the dimension of the enthesophytosis. We also evaluated the placebo effect by comparing one group of patients who underwent treatment with another group in whom the treatment was simulated.

ESWT proved effective in reducing the painful symptoms, and the reduction in pain seen at the end of treatment was maintained over the following three months. Furthermore, it was noted that the inflammatory oedema, assessed by sonography, which did not appear modified to any significant extent at the end of the treatment, was reduced to a significant degree one month later. In $30 \%$ of cases we saw a reduction in the enthesophytosis $>1 \mathrm{~mm}$, though this reduction was not significant compared with the control group. In the control group, no significant changes in pain levels, in the inflammation oedema, or in the dimensions of the enthesophytosis were seen. 
Our results confirm that the presence and size of bony spurs do not correlate with clinical symptoms $\mathrm{s}^{15}$ and that ESWT can, in our opinion, be considered the best treatment for painful heel with heel spurs, owing to its lack of side effects and because it is a repeatable and non-invasive treatment without anaesthesia.

1 Rompe JD, Hopf C, Nafe B, Burger R. Low-energy extracorporeal shocks wave therapy for painful heel: a prospective controlled single-blind study. Arch Orthop Trauma Surg 1996;115:75-9.

2 Bordelon RL. Subcalcaneal pain. A method of evaluation and plan for treatment. Clin Orthop Relat Res 1983;177: and plan

3 Maier M, Steinborn M, Schmitz C, Stabler A, Kottler S, Pfahler M. et al. Extracorporeal shock wave application for chronic plantar fasciitis associated with heel spur: prediction of outcome by magnetic resonance imaging. J Rheumatol 2000;27:2455-62

4 Sobel E, Levitz SJ, Caselli MA. Orthoses in the treatment of rear foot problems. J Am Podiatr Med Assoc 1999;89:22033.

5 Leroux JL. Tendinopathies calcifiantes de l'èpaule. La Lettre du Rhumatologue 1999;254:32-6.
6 Melzack R. Recent concepts of pain. J Med 1982;13:14760.

7 Maier M, Durr HR, Kolher S, Staupendahl D, Pfaheer H, Refior HJ. Analgesic effects of low energy extracorporeal shock waves for the treatment of tendinosis calcarea, epicondylitis humeri radialis and plantar fasciitis. Z Orthop Ihre Grenzgeb 2000;138:34-8.

8 Gibbon WW, Long G. Ultrasound of the plantar aponeurosis (fascia). Skeletal Radiol 1999;28:21-6.

9 Fornage BB. Achilles tendon: ultrasound examination. Radiology 1986;159:759-64.

10 Haupt G. Use of extracorporeal shock waves in the treatment of pseudarthrosis, tendinopathy and other orthopedic diseases. J Urol 1997;158:4-11.

11 Valchanou VD, Michailov P. High energy shock waves in the treatment of delayed and nonunion of fractures. Int Orthop 1991;15:181-4.

12 Sukul K, Johannes EJ, Pierik E, van Eijck G, Kristelijn M. The effect of high energy shock waves focused on cortical The effect of high energy shock waves focused on cortion
bone: an in vitro study. J Surg Res 1992;53:110-16.

13 Loew M, Jurgowski W. Extracorporeal stoßwellenLoew M, Jurgowski W. Extracorporeal sto $\beta$ wellen-
lithotripsie bei tendinosis calcarea. Z Orthop 1993;131: 470-3.

14 Perlick L, Boxberg W, Giebel G. High energy shock wave treatment of the painful heel spur. Unfallchirurg 1998;101: 914-18.

15 Schafer U, Micke O, Glashorster M, Rube C, Prott FJ, spurs. Strahlenther Onkol 1995;171:202-6. 